\title{
Die Kirche vor dem Problemkomplex der neuen Medien
}

\author{
von Gerburg E. Vogt
}

Im Frühjahr 1980 stand auf dem Programm eines katholischen Bildungszentrums eine Tagung zum Thema: Wem dient das Kabelfernsehen? Möglichkeiten und Risiken einer neuen Medientechnik. Da sich zwar alle Referenten, jedoch nur zwei interessierte Teilnehmer eingefunden hatten, mußte die Veranstaltung mangels Beteiligung abgesagt werden.

Spätestens seit der Internationalen Funkausstellung 1979 in Berlin sind die neuen Medien zwar heiß umstrittene Objekte der öffentlichen Diskussion, doch die zum Teil euphorischen Erwartungen auf ein Wunderland technisch vermittelter Kommunikation scheinen in Skepsis, Angst und Technikfeindlichkeit umzuschlagen. In der „professionellen” Diskussion hat man sich an den Gedanken einer grundlegenden Veränderung des Medienbereichs durch informationsverarbeitende und informationsvermittelnde technische Systeme gewöhnt. Dem einzelnen Bürger scheinen diese Aussichten immer unheimlicher zu werden.

Es drängen sich Fragen ins Bewußtsein, auf die auch die „Professionellen” keine oder keine zufriedenstellenden Antworten parat haben. Was kommt da auf uns zu? Wer entscheidet über diese Entwicklung? Welche materiellen und sozialen Kosten entstehen daraus und wer hat sie zu tragen? Wie wird die Familie, das Berufsleben, die Freizeit von diesen Veränderungen betroffen? Kann sich der einzelne Bürger an den Entscheidungen, die den Weg für diese Entwicklungen ebnen sollen, beteiligen und eventuell auch mitbestimmen?

Eine psychologische Studie über die Einstellungen der Fernsehzuschauer zur weiteren Entwicklung des Mediums Fernsehen ${ }^{1}$ weist darauf hin, daß ein Teil der Diskussion im Kommunikationssektor über den Bürger hinweggeht. „Die Geläufigkeit der Worte und Begriffe hat zugenommen. Insgesamt ist echtes Wissen über die neuen Techniken aber immer noch nicht groß."2 Wachsende Unsicherheit besonders hinsichtlich der Folgeerscheinungen veranlaßt besonders die Bedächtigen unter den Befürwortern neuer Kabelkommunikationsformen, ins Lager der Gegner überzuwechseln ${ }^{3}$.

Als ein Indiz dafür könnte auch die nur zögernde Bereitschaft der Bevölkerung in Berlin und Düsseldorf sein, an den Bildschirmtexterprobungen der Deutschen Bundespost teilzunehmen. Nach dem Erfolg der Internationalen Funkausstellung in Berlin im August 1979 mit ihren Besucherzahlrekorden sind die vorgesehenen 2.000 Anmeldungen privater Haushalte für die Erprobung, die am 1. Juni 1980 in Berlin beginnen soll, noch nicht erreicht. Für den Feldversuch in Düsseldorf entstanden Schwierigkeiten, die einzelnen Bevölkerungsschichten in der Zahl der zu testenden Haushalte repräsentativ vertreten zu haben. „Das Interesse der Düssel-

Sr. Gerburg E. Vogt sac promovierte in München mit einer Arbeit „Kirchen und Fernsehanstalten: Entwicklungen, Konzepte, Programmrealisierung". Sie ist Lehrbeauftragte für Kommunikationswissenschaft an der Hochschule für Philosophie München Philosophische Fakultät SJ und leitet die Vorversuche zur Beteiligung der Kirche an Bildschirmtext für die Zentralstelle Medien der Deutschen Bischofskonferenz. 
dorfer, sich an dem zwölfmonatigen Versuch zu beteiligen, ist . . . nach wie vor gering ... ; die Post sucht noch dringend Düsseldorfer mit geringem Einkommen, die bei dem Versuch mitmachen. Voraussetzung für eine Teilnahme ist allerdings ein Spezialfarbfernseher (zum Preis eines normalen Gerätes) und ein Telefonanschluß."

Werden hier die Unterschiede zwischen Bedürfnis und Bedarf deutlich, auf den die Kommission für den Ausbau des technischen Kommunikationssystems in ihrem Telekommunikationsbericht ${ }^{5}$ hingewiesen hat? Schrumpfen die angegebenen Bedürfnisse nach zusätzlichen Dienstleistungen im Informations- und Kommunikationsbereich zur Zufriedenheit mit dem bereits Vorhandenen zusammen, sobald diese Zusätze nicht gratis vergeben werden? Zeigen diese Reserven bei den potentiellen Benutzern, daß Lücken im gegenwärtigen Informationsnetz nicht oder noch nicht spürbar sind und daher weder Bedürfnis noch Bedarf vorausgesetzt werden dürfen? Wie begründen die Landesregierungen und -parlamente ihre Zurückhaltung, bis sie schließlich die Durchführung von Feldversuchen oder Pilotprojekten doch noch rechtlich ermöglichen? Läßt diese - unter einer Reihe von Aspekten ratlos anmutende Situation den Schluß zu, daß die Innovationspolitik der Post, der Elektro- und Elektronikindustrie es ist, die die Bevölkerung in dieses Dilemma zwischen Bedürfnis und Bedarf gebracht haben?

„Niemand hat den großen, überzeugenden, richtungsweisenden Entwurf: Weder der Bund noch die Länder; weder die Parteien noch die großen gesellschaftlichen Gruppen. 'Medienpolitik' erweist sich als ein zunehmend verschlungenes Knäuel von Wünschen, Begehrlichkeiten, Abwehrhaltungen, Partei-Egoismen, Utopien, Machtergreifungs- und Machterhaltungsstrategien; als ein Gewusel, in dem mittlerweile selbst die Experten aus Politik, Recht und Technik kaum noch durchgehende Linien ausmachen können." 6 So charakterisiert der Vorsitzende des Deutschen Journalisten-Verbandes, Manfred Buchwald, die Situation angesichts der technischen Revolutionserwartungen im Kommunikationsbereich.

Wolfgang und Otmar Ernst prognostizieren, daß sich in der Spanne bis zum Jahr 2000 noch nicht soviel verändert haben wird, daß der Begriff 'Revolution' angebracht erscheint; ,nur daß wir eben mehr Fernsehprogramme empfangen können, daß eine ganze Menge Leute einen Videorecorder haben, daß mit den meisten Fernsehgeräten Videotext empfangen werden kann und die Leute es nicht tun, daß sich der Bildschirmtext zu einem fast semiprofessionellen Kommunikationssystem elitärer Zielgruppen entwickelt hat und daß man Bildplatten an jedem Zeitungskiosk kaufen kann"7.

Für den nicht eingeweihten Betrachter dieser Medienausstattung der kommenden Jahre ist das schon eine ganze Menge, und eben in dieser trockenen Selbstverständlichkeit der Aufzählung liegt die Überraschung. Was hat es mit all dem auf sich? Diskutieren wir gar nicht mehr über die in diesem Szenario vorkommenden neuen Telekommunikationsformen? Ist ihre Einführung bereits so gut wie nicht mehr abzuwenden? Gehören die aufgezählten neuen Möglichkeiten vielleicht gar nicht zu dem Komplex der „Neuen Medien”?

Es ist in der Tat zu fragen, ob wir mit Recht von „Neuen Medien”sprechen können, oder ob es sich in der gegenwärtigen Diskussion nicht vornehmlich um eine Weiterentwicklung bereits verfügbarer Techniken handelt, die mit dem Begriff „Neue 
Medien" überbewertet und überinterpretiert werden. Andererseits zeigten sich an den verschiedenen organisatorischen Schnittstellen, daß sich durch den im KtKBericht empfohlenen beschleunigten Ausbau bereits vorhandener Übertragungsnetze und die Nutzung der dadurch verfügbaren Informationsvermittlungskapazität die Medienlandschaft nicht nur um neue Möglichkeiten, sondern um neue Zuständigkeiten verändern wird.

Das Institut der deutschen Wirtschaft geht in einer graphischen Anordnung einiger neuer technischer Informations- und Unterhaltungsangebote von einer Dreiteilung der technischen bzw. kommunikationspolitischen Zuständigkeitsbereiche aus. Die Gliederung berücksichtigt den Privatbereich, den Fernsprechbereich und den Rundfunkbereich ${ }^{8}$.

In den Privatbereich fallen alle jene Träger von Information, die von jedermann (der sie sich leisten kann und will) im freien Handel erworben werden können. Dazu zählen an neuen Angeboten vor allem Videobänder und Bildschirmspiele sowie die dazugehörenden Geräte. Der audiovisuelle Markt für den Heimbereich ist durch die steigenden Absatzzahlen für Videorecorder in der Bundesrepublik Deutschland gerade erst interessant geworden. Der Senator für Wissenschaft und Forschung in Berlin, Peter Glotz, weist in einem Vortrag auf der medienpolitischen Fachtagung der SPD zum Thema „Die Medien der achtziger Jahre” auf diesen privaten Bereich hin, dem möglicherweise erhöhte Bedeutung zukommen wird. „Über Mikroprozessoren mit Speichern kann man seinen Fernsehapparat zu einem Heim-Terminal umgestalten; mit Bildplatte und Videorecorder kann man eine stand-alone-Technologie entwickeln, die in den nächsten zehn bis fünfzehn Jahren zwar für den durchschnittlichen Bürger zu teuer sein dürfte, auf die aber die Nachfrage gelenkt werden könnte, wenn andere Möglichkeiten blockiert sind. Es würde dann aller Wahrscheinlichkeit nach ein Cassettenmarkt entstehen, der die direkte Zulieferung von Programmen an den eigenen Haushalt organisierte."9

Eine Graphik des multinationalen Konzerns ITT verdeutlicht diese Funktionserweiterung des Heimfernsehgeräts, die in den nächsten Jahrzehnten denkbar ist. Gerade der Preisrückgang elektronischer Produkte, der in den vergangenen Jahren an den Taschenrechnern besonders deutlich geworden ist, dürfte ein Hinweis darauf sein, daß dieser private Bereich und seine zukünftige individuelle Ausstattung mit Informations- und Kommunikationsmitteln nicht unterschätzt werden darf. K. Haefner charakterisiert solche privaten bzw. persönlichen Informations- und Telekommunikationssysteme der, wie er meint, endachtziger Jahre: „Es existiert eine zentrale Bank für die globale Information (ab ca. 1985), aus der man billige Massenspeicherauszüge beziehen kann; aktuelle Information ist on-line aus dem Zentrum aktueller Information verfügbar, über dieses wird auch weitere Informationsverarbeitungskapazität zur Verfügung gestellt. Das persönliche Informationsund Telekommunikationssystem (PITS) erlaubt Sprachein- und ausgabe, die Rechenleistung liegt im Bereich heutiger mittlerer Rechenzentren. Eine breite Palette fertiger Programme zur Lösung vieler Probleme steht ständig zur Verfügung; die Umsetzung eines einfachen Problems in ein auf dem PITS lauffähigen Programm ist auch für den Laien kein Problem. Es gibt Programme für viele Probleme, die leistungsfähiger als ein durchschnittlich ausgebildeter Mensch sind ... Große textverarbeitende Programme erzeugen aus den aktuellen Nachrichten der Presseagenturen unter Berücksichtigung aller relevanten Daten zu einem aktuellen Ereignis 


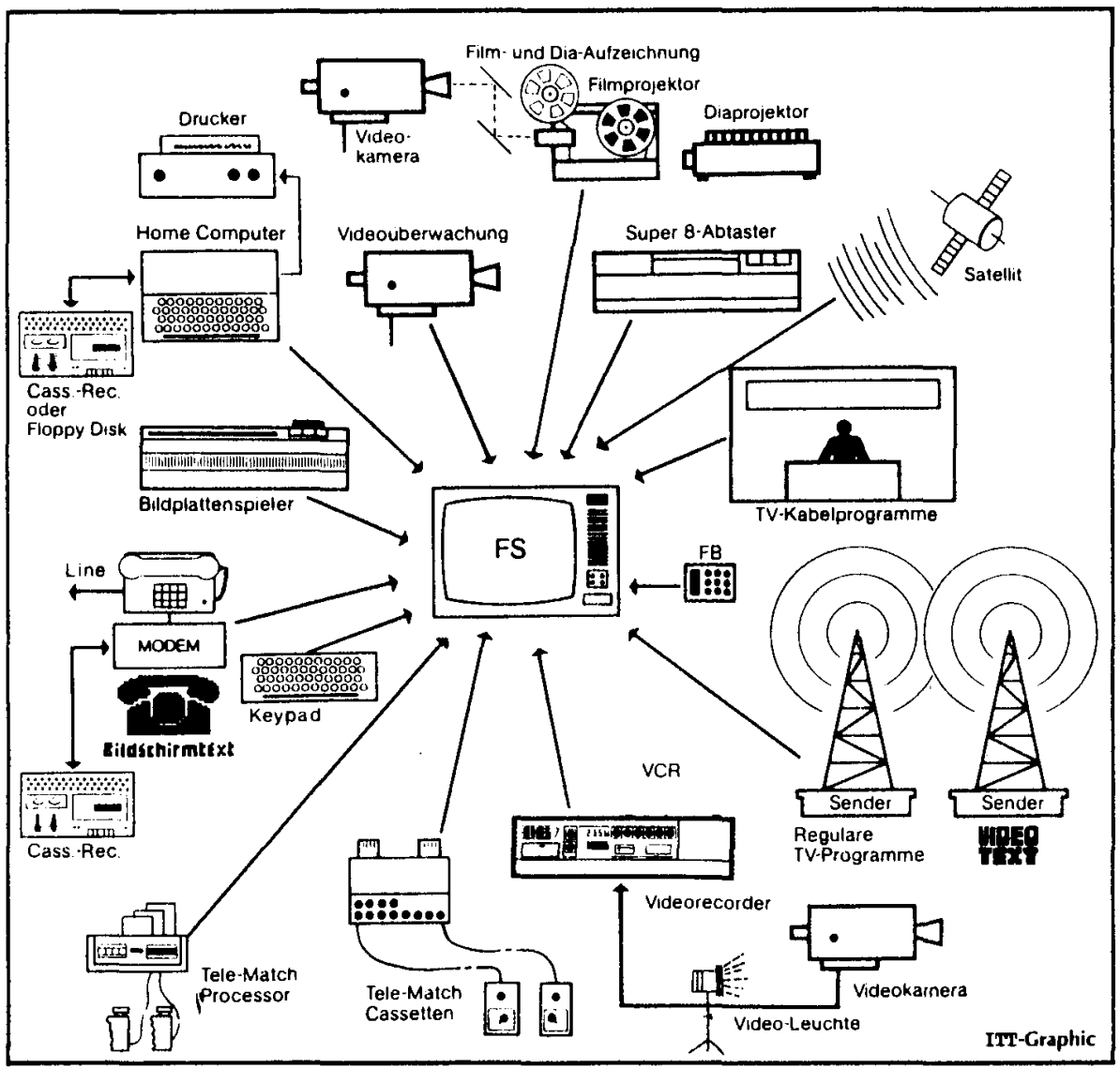

Das Farbternsehgerät als Terminal für zahllose Kommunikationstechniken

individuelle Artikel, die von den Redakteuren nur noch zum Zeitungsartikel überarbeitet werden ... Viele wichtige Vorgänge mit Behörden und Verwaltungen werden vom PITS unmittelbar abgewickelt, wobei es 'seinen' Menschen nur noch nach Daten befragt, die es selbst noch nicht kennt. Das PITS erlaubt die Unterhaltung in einer (relativ beliebigen) Fremdsprache (wobei die Partner sich allerdings noch auf einen nicht zu großen Wortschatz und recht einfache Sätze beschränken müssen), das PITS übersetzt simultan, beide Partner benutzen drahtlose Mikroplane und drahtlose Kopfhörer. Das PITS beantwortet einfache Fragen zu allen wichtigen Lebensfragen, wobei es allerdings den jeweils notwendigen Massenspeicher bzw. den Anschluß an das Zentrum aktueller Information braucht. Das Sprechschreiben ist mit jedem PITS möglich, allerdings werden nur noch wenige Drucker verwendet, da Information in aller Regel unmittelbar elektronisch umgesetzt und genutzt wird. Das PITS kann auf seiner Ausgabefläche oder auf einem großen 
Wandbildschirm jederzeit bildliche Information und Musik ausgeben, in bisher unbekannten Mischungen und Qualitäten, es kann die Sinne und Stimmungen in einem Maße verwirren, wie wir es heute nur nach Drogengebrauch kennen. Dies ist die Szene, auf deren Hintergrund wir die Frage nach der anthropologischen Akzeptanz einer 'Informierten Gesellchaft' stellen. Bedrängt oder zerstört dies alles den Menschen in seinem historisch entstandenen Selbstverständnis? Beginnt dieses Umweltproblem, den Menschen, seine Gefühlswelt, seine Stimmungen, seine Erkenntnis, seine Beziehungen und Grundwerte zu zerstören?"'l

Diese hier aufgezeigte Szenerie soll keine apokalyptischen Ängste hervorrufen, sondern lediglich zeigen, daß die Schritte, die heute durch die Errichtung von Datenzentralen bereits getan werden, im Zusammenhang weiterer Ausbaustufen gesehen werden müssen. Die individuelle Ausstattung mit Informations- und Kommunikationsmedien und der individuelle Zugriff zu einer Vielzahl von Unterhaltungs-, Informations- und Bildungsangeboten läßt mit Fug und Recht die Bezeichnung „Neue Medien” zu, auch für diejenigen Möglichkeiten, die jetzt noch im Bereich der Fernsprechdienste bzw. des Rundfunknetzes liegen und nur als zusätzliche Nutzungsmöglichkeiten vorhandener Übertragungskanäle anzusehen sind. Diese ergänzenden Dienste gewinnen sowohl in der Bearbeitung der dargebotenen Informationsteile als auch in ihrer Optik und in ihrem Gebrauch andere Qualitäten als die bisher gebräuchlichen Mittel.

In der öffentlichen Diskussion handelt es sich zumindest gegenwärtig nicht primär um private Mediotheken, sondern vielmehr um die Planung und Erprobung telekommunikativer Formen im öffentlichen Bereich, dazu gehören die Möglichkeiten, die im Fernsprech- und Rundfunknetz liegen, und vor allem die, die durch Verkabelungen mit Breitbandnetzen zusätzlich gegeben sind.

Die beiden Bereiche Fernsprechnetz und Rundfunknetz rücken im Zusammenhang mit den neuen Telekommunikationsformen nahe zusammen; nicht nur, weil beide Übertragungskanäle optisch sehr ähnliche Textinformationen vermitteln können, sondern auch weil im Zusammenhang ihrer Nutzung die Frage nach dem Rundfunk- bzw. dem Pressebegriff gestellt worden ist. Die Bezeichnung „Bildschirmzeitung" auf der Seite der Zeitungsverleger für ein System, das die Rundfunkanstalten Videotext nennen, ist sicher nicht ohne Hinweis auf die medienpolitisch ungeklärte Situation und die eigene Anwendungsabsicht zu verstehen. Der seit langem geführte Streit zwischen Zeitungsverlegern und Rundfunkanstalten um die Nutzung einer Lücke im Fernsehsignal zur Textübertragung soll hier nicht weiter kommentiert werden. Er zeigt nur, daß allen Beteiligten wohl bewußt ist, daß sich im Mediensektor mehr abspielt als das Angebot eines ,schlichten kleinen Zusatzdienst(es)"12.

Technisch kommt der Videotext folgendermaßen zustande: Die sogenannte vertikale Austastlücke - das ist der schwarze Balken, der auf dem Fernsehbildschirm sichtbar wird, wenn das Bild nicht richtig eingestellt ist und springt - ist bisher nur zum Teil genutzt worden. Die noch vorhandene Übertragungskapazität kann eine begrenzte Zahl von „Textseiten” transportieren. Eine solche Textseite besteht aus einer Kopf- und 23 Textzeilen mit bis zu 40 Zeichen pro Zeile. Diese 24 Reihen mit Schrift und einfachen Grafiken erscheinen auf dem Bildschirm des Heimfernsehers. Die Fernsehgeräte müssen mit einem eigenen Decoder ausgestattet sein, der die in der Austastlücke ankommenden Signale auf dem Bildschirm in Zeichen und Grafikteile umwandelt. Es dauert 0,23 Sekunden, bis eine Seite auf dem Fernseh- 
apparat sichtbar wird. In einem Übertragungskanal können acht mal 100 Texttafeln transportiert werden. Sie erscheinen zyklisch zu jeweils 100 Tafeln und können nur in diesem Verband abgerufen werden. Die Wartezeit zwischen Wahl und Darstellung auf dem Bildschirm dauert im Durchschnitt 12 Sekunden, maximal 24 Sekunden. Die gegenwärtig aufgenommenen Informationen beziehen sich vor allem auf aktuelle Nachrichten mehr in Form eines Schlagzeilendienstes, auf Programmhinweise und -voranzeigen, auf Veranstaltungstermine u.ä., Informationsteile, die in losem Zusamenhang mit dem Rundfunkprogramm stehen. Eine weitere Nutzung bietet die Untertitelung laufender Fernsehsendungen, was für gehörgeschädigte Menschen von besonderer Bedeutung sein dürfte.

Die Begrenztheit dieses zusätzlichen Dienstes liegt in seiner verhältnismäßig langen Aufbauzeit, in der festgelegten, nicht weiter vermehrbaren Zahl der Textseiten und in der Koppelung mit dem Fernsehsignal. Videotext ist also an den Sendebetrieb gebunden und kann zwar unabbängig vom Fernsehprogramm, aber nicht außerbalb desSendebetriebs abgerufen werden. Wie die konventionelle Fernsehübertragung ist auch der Videotext einbabnig. Es handelt sich also um einen Verteilerdienst, der keine Interaktion zwischen Teilnehmer und Zentrale zuläßt. Die Rundfunkanstalten wollen für Videotext keine eigenen Gebühren erheben. Der zusätzliche Informationsservice soll von den Fernsehgebühren mitfinanziert werden, was durchaus möglich sein dürfte. In England, wo die ersten Entwicklungen gemacht wurden und das System unter der Bezeichnung Teletext eingeführt worden ist, hat sich gezeigt, $\mathrm{da} ß$ sechs- bis siebenhundert Videotext-Tafeln, die die BBC in ihren beiden Programmen anbietet, jährlich nicht mehr kosten als eine einzige FernsehspielProduktion ${ }^{13}$.

Ein optisch dem Videotext zum Verwechseln ähnliches System bietet die Deutsche Bundespost seit Frühjahr 1978 potentiellen Informationslieferanten zu internen Vorversuchen an. Der Bildscbirmtext (nicht zu verwechseln mit der Bildschirmzeitung) wird im Telefonnetz übertragen und benutzt ebenfalls den Bildschirm des Heimfernsehers als Sichtgerät. Die Decoder der Farbfernsehgeräte der neuesten Generation können in der Regel sowohl Videotext-als auch Bildschirmtext-Signale verarbeiten.

Nachdem der KtK-Bericht den bevorzugten Ausbau vorhandener technischer Kommunikationssysteme empfohlen hatte, begann von seiten der Deutschen Bundespost eine intensive Werbung um private Telefonkunden. In der Zwischenzeit besitzen $70 \%$ der privaten Haushalte in der Bundesrepublik Deutschland einen Fernsprechanschluß. Der Trend sei immer noch ansteigend - so zumindest die Auskunft der Presseabteilung im Bundespostministerium vor der Abschaffung des Mondscheintarifs. Die Telefondichte und die Fernsehdichte sind Voraussetzungen für eine erfolgreiche Einführung von Bildschirmtext für die Gesamtbevölkerung.

Die bei Videotext angeführten Einschränkungen gibt es für Bildschirmtext nicht. Die Informationen, die nicht von der Post, sondern von einem breiten Spektrum von Informationslieferanten bereitgestellt werden sollen, sind aus einer Datenzentrale abrufbar. Die Kapazität solcher Datenspeicher ist praktisch unbegrenzt, zumal bei dem am 1. Juni 1980 in Düsseldorf und Neuss beginnenden Feldversuch und der Erprobung in Berlin zum ersten Mal auf der Welt ein sogenannter Rechnerverbund praktiziert werden soll. Dadurch soll dem Bildschirmtext-Teilnehmer die Möglichkeit geboten werden, Informationen direkt aus dem angebotenen Datenspeicher, 
z.B. einer Versandhandelsfirma, Informationen abzurufen und gleichzeitig mit Hilfe einer Fernbedienungstastatur die gewünschten Artikel zu bestellen.

Die Post hofft, daß Bildschirmtext ein weitverbreiteter, interessanter Fernmeldedienst für jedermann wird, vor allem auch, weil er Interaktionsmöglichkeiten zwischen Teilnehmer und Zentrale oder zwischen Teilnehmer und Teilnehmer zuläßt. Diese einfachste Form des Rückkanals ermöglicht frei formulierte schriftliche Mitteilungen an andere Benutzer. Dieser kann eine solche Benachrichtigung auf seinem Heimfernseher ablesen. Zum Schreiben solcher Texte ist eine Tastatur mit den Buchstaben des Alphabets notwendig. Bei vorbereiteten „Formularen” z.B. für eine Warenbestellung, eine Tischreservation, der Bitte um einen Rückruf u.ä. genügt eine Fernbedienung mit einigen Funktions- und Zahlentasten.

Neben der sebr hohen Kapazität an sogenannten „Bildschirmtext-Seiten”, des von einem Sendebetrieb unabhängigen Übertragungskanals und der Dialogfähigkeit des Systems Bildschirmtext, ist die selektive Auswahl aus dem vorhandenen Textangebot ein weiterer positiver Unterschied zum Videotext. Bei Bildschirmtext ist der unmittelbare Zugriff z.B. zur Seite 815 möglich, ohne dafür Wartezeit in Kauf zu nehmen. Sobald die betreffende Seitennummer auf der Tastatur eingegeben ist, baut sich die Bildschirmtext-Seite auf. Allerdings ist auch der Bildschirmtext an die begrenzte Zabl von Zeichen pro Seite gebunden. Es sind wie bei Videotext maximal 960 Buchstaben oder graphische Elemente pro Seite möglich. Dies macht eine kurze, präzise und leicht überschaubare Informationsdarbietung unumgänglich. In den übrigen schmalbandigen Netzen sind weitere neue Dienste bereits einsatzfähig, auf die ich hier nicht weiter eingehen will. Angebote wie Fernkopieren oder andere Arten von Festbildübertragungen werden vor allem für geschäftliche Nutzer und deren Bürokommunikation Bedeutung gewinnen.

Das besondere „catch-word” in der Diskussion um die zukünftige Medienlandschaft ist vor allem der Begriff Kabelfernsehen. Dabei liegt der Akzent vor allem auf dem zweiten Teil des Wortes, auf dem Stichwort „Fernsehen”. Dieses wiederum erweckt Assoziationen wie ,mehr Programme”, ,sex and crime”, „Droge für Kinder" u.ä. Ob unter dem Reizwort Kabelfernsehen an sechs, vierundzwanzig oder dreissig Kanäle gedacht wird, hängt von den Wünschen, Vorstellungen und dem Realitätssinn oder von dem Optimismus bzw. Pessimismus der Diskutierenden ab. Während für die einen mehr Programme gleichbedeutend mit einer weiteren Verflacbung der Inhalte ist, erwarten die anderen mehr Bürgernähe durch lokale oder regionale Informationen, mehr Lernangebote, mehr Bildungsprogramme. Während die einen die Wettbewerbsfähigkeit der deutschen Industrie auf dem Weltmarkt bereits erheblich geschwächt sehen, betonen die anderen, daß noch genügend Zeit sei, um die kommunikationstechnischen Strategien zu entwickeln, die notwendig sind, um die neuen Technologien sozial verantwortlich anzuwenden und zu organisieren.

Zum gegenwärtigen Zeitpunkt sind 8 Millionen Haushalte an Gemeinschaftsantennenanlagen angeschlossen. Sowohl der private als auch der durch die Deutsche Bundespost durchgeführte Ausbau eines breitbandigen Kabelnetzes erhöht sich in der Bundesrepublik Deutschland jährlich um $10 \%$.

Die Übertragung von Fernseh- und Hörfunkprogrammen über ein Netz von Kabeln, die von Zentralstellen ausgehen und zu den Steckdosen der einzelnen Teilnehmer führen, war zunächst ein Thema zur Versorgung benachteiligter Gebiete. 
Fernsehteilnehmer, die im Funkschatten von Bergen oder Hochhäusern wohnen, sollten die vorhandenen Rundfunkprogramme mit verbesserter Qualität empfangen können. Darüber hinaus bieten sich solche ohnehin notwendigen Netze dazu an, zusätzliche Programme, z.B. die Regionalprogramme der Rundfunkanstalten, die Programme der angrenzenden deutschsprachigen Länder, einzubeziehen. Die anfänglliche Fixierung auf Fernsebübertragungen hat die übrigen Nutzungsmöglichkeiten, die ein solches Kabelnetz bietet oder bieten könnte, außer acht gelassen. In der Zwischenzeit scheinen sich die geplanten Pilotprojekte in Berlin, Dortmund, München und Ludwigshafen/Mannheim so mit Diensten überfrachtet bzw. in solchen finanziellen Dimensionen zu befinden, da $B$ eine Realisierung lediglich für Ludwigsbafen in absehbarer Zeit erwartet werden kann. Was hier jedoch über ein Mehr an Fernsehprogrammen und eventuell einigen lokal bzw. regional orientierten Inhalten, die von privaten Anbietern produziert werden, den 20.000 vorgesehenen Haushalten an zusätzlichen Dienstleistungen auf den zwölf Kanälen mit Fernsehbreite angeboten wird, ist noch nicht deutlich geworden. Vermutlich werden Textvermittlungen möglich gemacht und der Anreiz des Offenen Kanals als Forum für die verschiedenen Gruppen im Verkabelungsnetz. Möglichkeiten, die für benachteiligte Menschen, z.B. Gehörgeschädigte, in einer solchen Region getestet werden könnten, müssen erst noch überlegt und eingebracht werden. Denn gerade Behinderte haben ein Recht darauf, die Hilfen, die die Technik anbietet, als Ausgleich für viele Benachteiligungen zu erhalten.

Ganz selten wird in der Argumentation um neue Kommunikationstechnologien auf sozial Benachteiligte hingewiesen. Es gibt viele Gruppen, denen durch technische Vermittlung wirksame Hilfe bzw. spezielle Dienste geleistet werden könnten, ohne daß die zwischenmenschliche Anteilnahme gleich auf dem Spiel stünde; ob es sich um schriftliche oder visuelle Interaktion zwischen Gehörgeschädigten handelt, um Sprachprobleme von Gastarbeitern und ihren Familien, um Umschulungsprogramme für Arbeitnehmer, um Bereitstellung rasch verfügbaren Materials für Gruppenarbeit in Erziehung, Schule oder anderen Bildungsbereichen. „Der Phantasie sind kaum Grenzen gesetzt, aber dem technisch Machbaren steht das wirtschaftlich Vertretbare gegenüber."14

Das ist sicher richtig. Nur manchmal, vielleicht sogar recht oft, spielen die Summen keine Rolle, obwohl der Zweck, dem sie dienen, durchaus als „sozial-unerwünscht”15 eingeordnet werden könnte. Hier kommt nun, am Ende dieses Problemkomplexes, die Position der Kirche.

Vorsichtig und grundsätzlich beginnt die Stellungnahme des Katholischen Büros in Mainz zum Referenten-Entwurf für ein Landesgesetz über die Durchführung eines Modellversuches mit Breitbandkabel, abgegeben vor dem Kulturpolitischen Ausschuß des Landtages Rheinland-Pfalz. Nach Auffassung der Katholischen Kirche hätten alle sozialen Kommunikationsmittel die Möglichkeit und Chance, das Zusammenleben der Menschen zu fördern. „Die Katholische Kirche neigt nicht von vornherein zum Kulturpessimismus. Das bedeutet allerdings nicht, daß sie einem blinden Fortschrittsglauben huldigt, schon gar nicht hinsichtlich einer nicht zuvor gründlich geprüften generellen Einführung des Kabelfernsehens." 16 Bei einer solchen Überprüfung wird für einen offenen Ausgang plädiert, auf die Rückholbarkeit der Versuche sei größter Wert zu legen. Ob das realistisch ist, bei 20.000 geplanten Teilnehmern? Ob nicht doch der Vorschlag des Leiters der Zentralstelle Medien der 
Deutschen Bischofskonferenz, Wilhelm Schätzler, bedenkenswert wäre, nämlich in bereits bestehenden Kabelverbänden Einzelerprobungen durchzuführen ${ }^{17}$ ? Warum sollte nicht das Schwarzwalddorf Unterkirnach mit seinen 2.400 Einwohnern, die seit dem 1. Oktober 1979 ihre Fernsehprogramme auf dem Kabelweg erhalten, ein Exempel für ein lokales Programm oder den Offenen Kanal statuieren können für andere ländliche Gebiete?

Ein weiterer neuralgischer Punkt in allen Kommunikationszusammenhängen ist die Repräsentanz der vorhandenen Kommunikationspartner und ihrer Beteiligung. Auf die Berücksichtigung aller kommunikativen Interessen, ohne Dominanz des Staates oder einseitiger Machtgruppen, weisen beide oben angeführten Stellungnahmen hin.

Neue Entwicklungen, deren Vor- und Nachteile nicht spekulativ, sondern im sozialen Umfeld beurteilt werden sollen, bringen neben der Faszination auch Befürchtungen zutage. Viele Befragte im Bereich eines Pilotprojekts gaben an, daß sie die Angebote gar nicht haben wollten, und lassen zur „Stunde des Tests” dennoch „Akzeptanz” erkennen. „Viele Interviewte waren sich dessen bewußt, daß sie die neuen Medien nutzen würden, wenn sie einmal da sind. Sie ließen aber zugleich erkennen, das bedeute nicht Zustimmung." 18 Nicht nur Unsicherheit, sondern ein deutliches Ohnmachtsgefühl, den Gang der Dinge nicht beeinflussen zu können, wird daraus deutlich. Gerade die Berücksichtigung der Wünsche und Interessen der Betroffenen sprechen die Stellungnahmen immer wieder an, ob es sich um die Art der Verkabelung oder die Struktur der lokalen Programmangebote handelt.

In einem relativ begrenzten Medium, für die Erprobung von Bildschirmtext, sind beide Kirchen als Informationsanbieter beteiligt. Gerade dieses System ermöglicht es, das breite soziale und pastorale Angebot der unterschiedlich strukturierten Ortskirche darzustellen und auf die verschiedenen Hilfsmöglichkeiten hinzuweisen. Allerdings erscheint gerade für dieses relativ leicht erreichbare Informationssystem eine Blockade durch die Telefongebühren vorhanden zu sein. Zwar steigt täglich die Zahl der Telefonkunden auch aus den einkommensschwächeren Schichten der Bevölkerung, doch der Zeittakt bei der Gebührenberechnung bzw. die Telefongebühr überhaupt könnte z.B. manchen alten Menschen davon abhalten, sich über Gottesdienstzeiten oder den Altennachmittag zu informieren.

Doch es sind nicht nur die organisatorischen, finanziellen oder technischen Fragen, die von Bedeutung sind, obwohl sie wesentliches zur kirchlichen Zielvorstellung, dem Dienst an der menschlichen Kommunikation, beitragen. Alle, die sich über die Vermittlung von Mitteilung und Kommunikation Gedanken machen, sollten sich der unterschiedlichen Vermittlungsformen bewußt werden und die Bedürfnisse der verschiedenen Kommunikationspartner und ihre Erwartungen an die „engagierte und kritische Mitarbeit der Kirche"19 erwägen. 


\section{Anmerkungen}

1 Albrecht Müller: Einstellungen der Fernsehzuschauer zur weiteren Entwicklung des Mediums Fernsehen, „Media-Perspektiven”, Frankfurt/Main, 3: 1980, S. 179-186.

2 Ebd. S. 181.

3 Ebd. S. 182.

4 Düsseldorfer Nachrichten vom 26.2.1980.

5 Kommission für den Ausbau des technischen Kommunikationssystems (Hrsg.): Bedürfnisse und Bedarf für Telekommunikation, Bonn 1976, Anlageband 1.

6 Manfred Buchwald: Thesen zur Medienpolitik, in: ,journalist” 5: 1980, S. 18-19 und 30.

7 Wolfgang Ernst/Otmar Ernst: Kriterien der Medienselektion und die Chancen für neue Medien, S. 206, in: Eberhard Witte (Hrsg.): Telekommunikation für den Menschen, Berlin, Heidelberg, New York 1980, S. 185-206.

8 ZAW-service Nr. 82, Dezember 1979, S. 16.

9 Peter Glotz: Die Medien der achtziger Jahre, in: ,journalist” 2: 1980, S. 41-47.

10 Entnommen aus: „journalist” 5: 1980, S. 11.

11 K. Haefner, Aufbruch in die informierte Gesellschaft? - Konsequenzen der Informationstechnik für die 80er Jahre, zitiert nach dem unveröffentlichten Entwurf, Juli 1979, Kapitel 4, Abschnitt 1-2.

12 Jens Brüning: Medienpolitischer Sündenfall oder schlichter kleiner Zusatzdienst?, in: „Funk-Korrespondenz” Nr. 19, 7. Mai 1980, S. 4.

13 O.Vf.: Die Kosten, in: „journalist” 5: 1980, S. 8-11.

14 Wolfgang Kaiser: Vorwort zu „Kabelfernsehen”, Anlageband 5 zum Telekommunikationsbericht, Bonn 1976, S. V.

15 Hansjörg Bessler: Brutalität im Fernsehen, in: Dieter Prokop (Hrsg): Massenkommunikationsforschung, Band 2, Frankfurt/Main 1973, S. 272.

${ }^{16}$ Katholisches Büro Mainz, Stellungnahme zum Referenten-Entwurf für ein Landesgesetz über die Durchführung eines Modellversuches mit Breitbandkabel - Stand 13. November 1979 - abgegeben vor dem Kulturpolitischen Ausschuß des Landtages Rheinland-Pfalz am 11. Dezember 1979, unveröffentlichtes Manuskript, S. 1.

17 Zentralstelle Medien der Deutschen Bischofskonferenz: Neue Techniken im Medienbereich, Öffentliche Anhörung des Hauptausschusses des Landtags Nordrhein-Westfalen am 16. und 17. Januar 1980, unveröffentlichtes Manuskript, S. 3.

18 Albrecht Müller: Einstellungen der Fernsehzuschauer zur weiteren Entwicklung des Mediums Fernsehen, „Media-Perspektiven”, Frankfurt/Main, 3: 1980, S. 179-186.

19 Katholisches Büro Mainz, S. 3. 


\section{SUMMARY}

New forms of telecommunications such as videotext, Ceefax, Cable TV, already being developed in European countries, are under consideration and discussion in the Federal Republic of Germany. In tests and pilot projects, efforts are being made to assess the sociological and economic consequences of the new technology. The Catholic Church shares in these experiments, with the aim of using the opportunities they provide to develop a better sense of 'togetherness' on regional and local levels. In order to minimise risks it is important to involve the people concerned and arouse their critical awareness of the tests and the progress of the project. Along with the opportunity of using new technology is the chance of new and better exchange of information. Some 'de-mythologising' is needed to simplify the complicated terminology and present economic, technical, political and sociological arguments in clear and decisive terms.

\section{RÉSUMÉ}

Des formes nouvelles de télécommunication telles que le texte vidéo, le texte-écran, la communication à ruban large - déjà en partie réalisées dans d'autres pays européens et extra-européens - sont, en République Fédérale d'Allemagne, l'objet de discussions publiques. On doit en plus tester les conséquences mondaines, économiques et sociales à l'aide de recherches, à savoir de projets pilotes. L'Eglise catholique collabore à différents essais préliminaires. Son but escompté: utiliser la chance de nouveaux moyens de télécommunication pour encourager la vie en commun de gens dans le domaine local et régional. Pour en diminuer les risques, il est nécessaire d'accompagner les essais de façon engagée et critique et de faire participeraux décisions les citoyens concernés. Le devoir d'information des concernés va de pair avec la question de l'utilisation des nouveaux media. Il faut détruire le fatalisme d'une technique compliquée et les arguments sociaux, politiques, techniques et économiques qui lui font face et auxquels on ne peut à peine échapper par un concept de décision dont on peut socialement répondre.

\section{RESUMEN}

En Alemania Federal son objeto de pública discursión nuevas formas de comunicación, como el videotext, la prensa visualizada y la comunicación de banda ancha -- ya realidad en parte en otros países europeos y no europeos. En proyectos piloto deben chequearse sus repercusiones económicas y sociales. La iglesia católica colabora en los diversos tests. Su meta es aprovechar las posibilidades de los nuevos medios de telecomunicación para promover la convivencia humana a nivel local y regional. Para disminuir los riesgos es necesario un chequeo comprometido y crítico y la participación en las decisiones de los ciudadanos afectados. Paralelamente a la cuestión de la utilización de nuevos medios, se encuentra la misión de informar a los efectados. El fatalismo frente a una técnica complicada y la falta de argumentos económicos, técnicos, políticos y sociales para rebatirla debe superarse mediante un concepto para decisiones sociales responsables. 